\title{
Der Niedersachsen-Weg - Tarifregelungen, Einführungsprozesse und Wirkungen des ERA
}

\author{
Martin Kuhlmann \\ Hans Joachim Sperling
}

\begin{abstract}
Die Hauptphase der Einführung der neuen Entgeltrahmentarifverträge (ERA) in der Metall- und Elektroindustrie ist in den Betrieben mittlerweile abgeschlossen, was erste Bilanzierungen ermöglicht. Kann ERA als gelungene Nagelprobe für die Zukunftsfähigkeit des Flächentarifvertrags gelten? Die Antworten müssen differenziert ausfallen. Da die tariflichen Regelwerke und die Orientierungen der Akteure Unterschiede aufweisen, ist dies auch für ihre Umsetzung und Effekte zu erwarten. Begründen die besonderen Tarifregelungen sowie die Erfahrungen und Orientierungen der Tarifparteien in Niedersachsen einen spezifischen Weg der ERA-Umsetzung? Welche Varianten der Einführungsprozesse und welche Wirkungen lassen sich feststellen?
\end{abstract}

\section{Einleitung}

Mit den zwischen 2003 und 2005 vereinbarten Entgeltrahmentarifverträgen (ERA) haben sich die Tarifparteien der Metallund Elektroindustrie nach langjährigen Verhandlungen auf ein tarif- und gesellschaftspolitisches Reformvorhaben verständigt, das auf eine zeitgemäße Neubewertung von Arbeit und Leistung zielt und - als ,größte Tarifreform der Nachkriegsgeschichte " und „Jahrhundertprojekt" tituliert - mit beachtlichem publizistischen und organisatorischen Aufwand in die Phase der Umsetzung gestartet ist (Huber/ Schild 2004; Schulz 2004; Manthey/Meine 2004; Sadowsky 2004; Beraus 2004; Reichel et al. 2005; Brunkhorst et al. 2006). Die Modernisierung bislang geltender Tarifnormen erfolgt einerseits über die Anwendung einer einheitlichen, anforderungsbezogenen Bewertungssystematik, mit der die bisherige Trennung von Arbeiter- und Angestelltentätigkeiten aufgehoben wird und den technischen, organisatorischen und sozialen Veränderungen der Arbeitswelt Rechnung getragen werden soll. Andererseits beinhaltet ERA eine Neuordnung von Entgeltgrundsätzen und -methoden und damit eine Neujustierung des Verhältnisses von Entgelt und Leistung. Bei ERA handelt es sich um ein ambitioniertes Reformprojekt, bei dem es angesichts abnehmender Tarifbindung und Verpflichtungsfähigkeit der Tarifparteien auch um die Frage der Stabilisierung des Flächentarifvertragssystems geht (Streeck/Rehder 2003; Huber et al. 2006; Bispinck 2007). Von der Umsetzung der Entgeltrahmentarifverträge wur- den deshalb auch Aufschlüsse über die Reformfähigkeit des Flächentarifvertrags erwartet. Von Seiten der Arbeitgeberverbände wie der IG Metall wurde die betriebliche ERA-Umsetzung mit erheblichem Qualifizierungs- und Unterstützungsaufwand (Schulungen, Erfahrungsaustausche, Beratungsangebote) begleitet. Obgleich die zentralen Grundlinien der ERA-Abkommen weitgehend identisch sind, zeichnen sich die jeweiligen Tarifverträge durch eine Reihe von Unterschieden aus, die den regionalen Besonderheiten Rechnung tragen.

Ausgehend von Zwischenergebnissen eines noch laufenden Forschungsprojektes zum ERA im Tarifgebiet Niedersachsen sollen im Folgenden erste Befunde zur ERA-Umsetzung vorgestellt werden. ${ }^{1} \mathrm{Oh}-$ ne an dieser Stelle in einen systematischen Vergleich der unterschiedlichen ERA-Tarifverträge einzutreten (vgl. hierzu die synoptische Darstellung auf der CD-ROM in Brunkhorst et al. 2006 sowie Reichel 2005), werden angesichts der regionalen Unterschiede zunächst einige Besonderheiten der tarifvertraglichen Regelungen in Niedersachsen dargestellt, die, so die These, insbesondere für den Verlauf des Umsetzungsprozesses nicht ohne Folgen sind (Abschnitt 2). Basierend auf Fallstudien stellen wir anschließend dominante Muster der ERA-Einführungsprozesse in Niedersachsen vor (Abschnitt 3), bevor in einem nächsten Schritt ausgewählte, vorläufige Befunde zu den Wirkungen von ERA präsentiert werden (Abschnitt 4). In einer abschließenden (Zwischen-)Bilanz (Abschnitt 5) werden dann einige Einschätzungen zur Bewertung der bisherigen ERAUmsetzung im Tarifgebiet Niedersachsen formuliert. Der Grundtenor der bisher vorliegenden Untersuchungsergebnisse lässt es gerechtfertigt erscheinen, von einem „Niedersachsen-Weg" zu sprechen, dessen WegMarkierungen in den Tarifregelungen enthalten sind, die Erfahrungen und Orientierungen sowie strategische Überlegungen der Tarifakteure ausdrücken.

\footnotetext{
Das Projekt wurde von der Hans-Böckler-Stiftung gefördert und vom Soziologischen Forschungsinstitut (SOFI) an der Universität Göttingen durchgeführt. Empirische Basis des vorliegenden Textes (Stand: Herbst 2008) sind Expertengespräche mit für ERA zuständigen hauptamtlichen Gewerkschaftsvertretern auf Verwaltungsstellenebene und in der Bezirksleitung sowie mit den ERA-Experten des Arbeitgeberverbandes NiedersachsenMetall und Kurzfallstudien in 22 Betrieben, die ERA fast durchweg bereits eingeführt haben. In den Fallstudien wurden in der Regel Gespräche mit den zuständigen Betriebsräten und den Verantwortlichen des Betriebes sowie Begehungen durchgeführt und betriebliche Unterlagen und Daten gesichtet. Teilweise berücksichtigt wurden Ergebnisse aus Intensivfallstudien (Interviews mit Bereichsleitern, Gruppendiskussionen mit ERA-Betroffenen). Im Rahmen des Forschungsprojektes noch geplant ist eine schriftliche Befragung aller Betriebe, die in den Geltungsbereich des ERA in Niedersachsen fallen.
}

Martin Kuhlmann, Dr., Soziologe, Mitarbei ter am Soziologischen Forschungsinstitut an der Universität Göttingen (SOFI). Arbeitsschwerpunkte: Industrie- und Arbeitssoziologie, Industrielle Beziehungen. e-mail: martin.kuhlmann@sofi.unigoettingen.de

Hans Joachim Sperling, Dr., Dipl.-Soziologe, Mitarbeiter am SOFI. Arbeitsschwerpunkte: Industrie- und Arbeitssoziologie, Industrielle Beziehungen. e-mail: joachim.sperling@sofi-unigoettingen.de 


\section{ERA in Niedersachsen: Kontinuitäten und Wandel}

Bei der Beschreibung und Bewertung der Arbeitstätigkeiten, die bei ERA in jedem Fall neu erfolgen müssen, orientiert sich der niedersächsische Tarifvertrag an dem bisher schon praktizierten Verfahrensgrundsatz der summarischen Arbeitsbewertung. Man verzichtete auf die Einführung grundsätzlich neuer Verfahren und damit auf einen Weg, den die Tarifparteien in BadenWürttemberg und in Nordrhein-Westfalen eingeschlagen haben. Mit dem Festhalten an einer etablierten Bewertungssystematik, die sich aus Sicht beider Tarifvertragsparteien im Großen und Ganzen bewährt hat, bleibt der Qualifizierungs- und Umstellungsaufwand in Niedersachsen begrenzter. Gleichwohl distanzieren sich beide Tarifseiten von einer rein rechnerischen Regelüberleitung, bei der die Beschäftigten aufgrund ihrer bisherigen Eingruppierung in eine neue Entgeltgruppe eingestuft werden. Die Notwendigkeit, alle Beschäftigten auf der Basis von anzufertigenden Tätigkeitsbeschreibungen neu einzugruppieren, wollte man den Betrieben nicht ersparen, da erst auf dieser Grundlage die Zielsetzung einer Neuordnung der betrieblichen Eingruppierungsstrukturen zu erreichen war.

Während in der Verfahrensfrage „Summarik“ Einigkeit herrschte, war der Verzicht auf einen Katalog von tariflichen Richt- bzw. Niveaubeispielen, wie er zunächst in Baden-Württemberg vereinbart und dann von einigen Tarifregionen in modifizierter Form übernommen wurde, Ergebnis einer Nichteinigung. Nachdem die Übernahme der Niveaubeispiele aus Baden-Württemberg abgelehnt und Verhandlungen über eigene Texte begonnen wurden, gelang es zunächst lediglich, Tarifbeispiele für den Bereich Zerspanung zu verabschieden. Angesichts der von beiden Seiten als zäh und langwierig beurteilten Verhandlungen wurde dann jedoch entschieden, auf einen Katalog von Richtbeispielen zu verzichten. Offiziell standen damit den betrieblichen Akteuren keine tariflichen Richt- oder Niveaubeispiele als Orientierung für die Bewertung zur Verfügung - informell spielte ein von NiedersachsenMetall erstellter Katalog in einigen Betrieben aber durchaus eine Rolle. Von beispiel- haften Tätigkeitsnennungen im Gehaltsbereich abgesehen, enthielt allerdings auch der vorherige Tarifvertrag keine Richtbeispiele.

Ebenfalls spezifisch für ERA in Niedersachsen sind zwei für den Einführungsprozess überaus bedeutsame tarifliche Vorgaben. Der Einführungszeitpunkt kann nicht einseitig vom Arbeitgeber festgelegt werden, sondern bedarf einer einvernehmlichen Regelung. Die Besitzstandsicherung ${ }^{2}$ der Einkommen erfolgt tarifdynamisch, sodass aus Arbeitgebersicht zumindest kurzfristig kein starker Anreiz besteht, im Rahmen von ERA-Eingruppierungen Personalkosten zu senken.

Ein erster Befund zu ERA in Niedersachsen lautet insofern, dass zentrale Regelungen sich durch einen eher konfliktdämpfenden Charakter auszeichnen und den betrieblichen Akteuren ein hohes Maß an Kooperation nahelegen. Während die Etablierung einer einheitlichen Entgeltsystematik einen weitreichenden Eingriff darstellt, ist das Ausmaß von Kontinuitäten bei den technischen Regelungen der Eingruppierung, bei Reklamationsverfahren sowie bei der Frage der Entgeltgrundsätze sehr viel größer. Typisch für die ERAEinführung im Tarifgebiet Niedersachsen ist, dass die Tarifvertragsparteien sich hier nicht in der Rolle von Treibern der Veränderung betrieblicher Entgeltlinien oder von Entgeltsystemen sehen. Beide Tarifvertragsparteien betonten zwar die Notwendigkeit einer neuen Eingruppierung aller Beschäftigten und erhofften sich von der Korrektur überkommener Eingruppierungen ein höheres Maß an anforderungsbezogener Gleichbehandlung. Im Mittelpunkt der Verbandsaktivitäten standen jedoch nicht möglicherweise zu erreichende Kostensenkungen oder Entgeltzuwächse, sondern es ging um eine fach- und sachgerechte Umsetzung der Regelungen des Tarifvertrags. Während andere Arbeitgeberverbände zur Steuerung und Kontrolle der ERA-Umsetzung teilweise in erheblichem Umfang zusätzliche Ressourcen mobilisierten und Verbandsingenieure rekrutierten, verzichtete NiedersachsenMetall bewusst hierauf und sah sich eher in der Funktion eines Dienstleisters, der sich auf die Bereitstellung von Informationsmaterial und Umsetzungshilfen sowie die Durchführung von Informations- und Erfahrungsaustausch konzentrierte und auf Anfrage den Mitgliedsfirmen mit Beratungsleistungen zur Verfügung stand, jedoch keinen Druck auf die Betriebe ausübte.

Auch von der zuständigen Bezirksleitung der IG Metall in Hannover wurden für die ERA-Umsetzung nur in eher beschränktem Ausmaß zusätzliche Personalkapazitäten bereitgestellt; auf der Basis bestehender Strukturen auf bezirklicher Ebene, in den Verwaltungsstellen und beim zuständigen Bildungszentrum (Sprockhövel) wurden jedoch vorhandene Ressourcen mobilisiert und fokussiert. Der tarif- und betriebspolitische Gestaltungsanspruch, der sich auf Seiten der IG Metall an ähnlichen Zielsetzungen (anforderungsbezogene Neu-Eingruppierung, Absicherung der bisherigen Entgeltniveaus, behutsame Modernisierung bestehender Entgeltsysteme ohne klare „Vorgaben“ hinsichtlich zu bevorzugender Lösungen) orientierte, wurde über ein breit angelegtes Informations-, Qualifizierungs- und Beratungskonzept aufgegriffen und umgesetzt. Durch die Einrichtung von Steuerkreisen auf den Ebenen des Bezirks und der Verwaltungsstellen, die Durchführung von mehrstufigen Schulungseinheiten (Ausbildung von ERAExperten als Multiplikatoren), betriebsnahen Erfahrungsaustausch sowie betrieblichen Schulungen und Beratungen (Inhouse-Seminare und Coaching-Angebote) wurden Wissen und Kompetenz bezogen auf Entgeltfragen bei gewerkschaftlichen und betrieblichen Funktionären neu ausgebildet und auf eine breitere Basis gestellt.

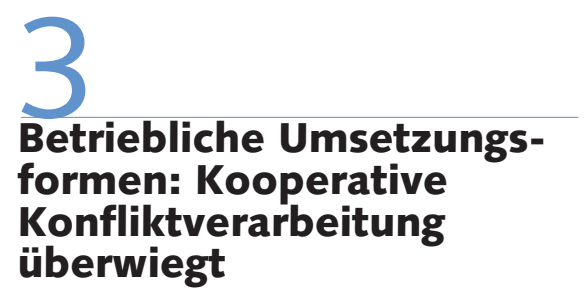

Die ERA-Umsetzung in Niedersachsen ist bislang vergleichsweise geräuscharm verlaufen und von Varianten eines Grundmusters der Konfliktpartnerschaft („ko-

\footnotetext{
2 Während die in anderen Tarifgebieten als „Überschreiter " bezeichneten Beschäftigten (Personen, die in der tariflichen Eingruppierung heruntergestuft worden sind, aber aufgrund einer Überleitungszulage keine Einkommenseinbußen haben) zukünftig teilweise auf Tariferhöhungen verzichten müssen, ist dies durch die tarifdynamisch gesicherten und nicht anrechenbaren Überleitungszulagen im Tarifgebiet Niedersachsen ausgeschlossen.
} 
operative Konfliktverarbeitung" (Weltz 1977)) geprägt. Die Betriebsparteien begreifen ERA als betriebliches Projekt, das Handlungs- und Gestaltungsspielräume ermöglicht, zugleich aber orientieren sie sich vergleichsweise eng an den tarifvertraglichen Vorgaben. Diese lassen ausreichend Spielräume und legen gleichzeitig eine kooperative Vorgehensweise nahe, schließen aber auch konflikthafte Zuspitzungen nicht aus. Insgesamt erweist sich die ERA-Umsetzung - wie in anderen Tarifgebieten auch - als zeitaufwendiger und komplexer als ursprünglich erwartet. Veränderungen bei Entgeltgrundsätzen und -methoden werden in der Regel vertagt, die Umsetzung richtet sich im Wesentlichen auf die Frage der Beschreibung und Bewertung der Arbeitstätigkeiten. In den meisten Betrieben, aber nicht durchgängig, werden zunächst Arbeitsbeschreibungen erstellt und zwischen den Betriebsparteien abgestimmt, bevor dann Eingruppierungen erfolgen. Unter Wahrung tarifvertraglich vorgegebener, in den meisten Betrieben durch Betriebsvereinbarungen jedoch verlängerter Beratungs- und Reklamationsfristen findet dann eine Information des Betriebsrates und der Beschäftigten über ihre Eingruppierung statt. Nachdem die strittigen Eingruppierungen (ggf. vorläufig) geklärt sind, tritt ERA dann zum vereinbarten Zeitpunkt in Kraft. Charakteristisch für die Einführungsprozesse ist, dass die betriebliche Praxis in der großen Mehrzahl der Betriebe in Punkten wie Information durch den Arbeitgeber sowie Mitwirkung beim Thema Eingruppierung über die im Tarifvertrag vorgesehenen Mindestanforderungen hinausgeht. Trotz vieler Detailunterschiede in der ERAUmsetzung lassen sich hierbei vier Grundtypen identifizieren. Entlang dem Grad der Konflikthaftigkeit finden sich kooperativkonsensuale, partiell konflikthafte und durchgängig konflikthafte Einführungsprozesse, wobei sich der erste Typ noch einmal in eine gemeinsame und eine auf getrennten Arbeitsgruppen basierende Vorgehensweise differenziert.

\subsection{KOOPERATIVE ERA-UMSETZUNG: GEMEINSAM ODER GETRENNT?}

Weitgehend konfliktfrei und konsensorientiert ist ERA in Betrieben umgesetzt worden, in denen die Betriebsparteien eine belastbare kooperative Zusammenarbeit schon in der Vergangenheit praktiziert und
ERA deshalb als gemeinsames Projekt verstanden und betrieben haben. Ein hohes $\mathrm{Maß}$ an Übereinstimmung herrschte in diesen Betrieben zudem bezogen auf die Auslegung und Anwendung des Tarifvertrags und den Einführungsprozess. Hinsichtlich der konkreten Vorgehensweise und der Arbeitsformen sind allerdings zwei sehr unterschiedliche Konstellationen und damit Typen der ERA-Umsetzung zu unterscheiden.

Als kooperativ-gemeinsam (Typ I, drei Betriebe) lässt sich ein Umsetzungstyp bezeichnen, bei dem die Betriebsparteien frühzeitig eine gemeinsame Arbeitsgruppe bilden, die den gesamten ERA-Prozess steuert und in der insbesondere die Arbeitsbeschreibungen und Eingruppierungen Schritt für Schritt gemeinsam erarbeitet und innerbetrieblich abgeglichen werden. Konkret kann es sich hierbei um einen langfristig angelegten Prozess handeln, in dem neben den ERA-Experten auch weitere betriebliche Akteure (insbesondere zuständige Bereichsleiter) sowie die Beschäftigten selbst eingebunden sind; es finden sich bei diesem Typ aber auch Beispiele für einen stark konzentrierten Arbeitsprozess „im kleinen Kreis“.

Der quantitativ mit neun Betrieben am stärksten in unseren Fallstudien vertretene Typ II (kooperativ-getrennt) zeichnet sich ebenfalls durch ein hohes Maß an Kooperation und ein konfliktpartnerschaftliches Grundverständnis aus, das Dissens in Einzelfragen nicht ausschließt und von unterschiedlichen Interessenlagen ausgeht. Auch in diesen Fällen legen die Akteure großen Wert auf eine korrekte Umsetzung des Tarifvertrags, zugleich sollen „Unruhe in der Belegschaft" sowie innerbetriebliche Konflikte vermieden werden. Bei diesem Umsetzungstyp arbeiten die Zuständigen der Unternehmensseite und des Betriebsrates jedoch zunächst getrennt in eigenen Arbeitsgruppen, bevor (Zwischen-)Ergebnisse dann in gemeinsamen Runden ausgetauscht und verhandelt werden.

Die Unterschiede zwischen diesen beiden Typen liegen vor allem in den betrieblichen Traditionen, im Bereich der Interessenvertretungsstile und dem Charakter der betrieblichen Arbeitsbeziehungen. In den Betrieben des Typ II wird insbesondere von Seiten der betrieblichen Interessenvertretung ein größeres Gewicht darauf gelegt, Eigenständigkeit gegenüber dem Management auch nach außen zu demonstrieren.

\subsection{KONFLIKTHAFTE ERA-UMSETZUNG}

Zwei weitere Typen der ERA-Umsetzung zeichnen sich demgegenüber durch größere Unterschiede in der Sichtweise bezogen auf den Einführungsprozess und die bei ERA angestrebten Ergebnisse aus. Folglich gibt es hier ein höheres Konfliktpotenzial. Teils spielen Konflikte in diesen Betrieben generell eine größere Rolle, häufig sind sie Folge eines höheren Anteils von Beschäftigten mit Überleitungszulagen, mitunter resultieren sie aber auch aus Versuchen des örtlichen Managements, unternehmensbzw. konzerneinheitliche Vorgaben umzusetzen. Beim Typ III, begrenzte Konflikte (sieben Betriebe), trägt der ERA-Prozess zwar immer noch Züge einer kooperativen Konfliktverarbeitung, in relevanten Punkten gibt es in diesen Betrieben jedoch erhebliche Meinungsverschiedenheiten und der Gesamtprozess wird nicht mehr in toto als von einem gemeinsamen Verständnis getragen gesehen. Zumindest teilweise begegnen sich die Betriebsparteien daher mit Misstrauen; die Unsicherheit, inwieweit sich die Gegenseite als sachlich-fachlich orientierter Verhandlungspartner erweist, wächst.

Auch im Tarifgebiet Niedersachsen gibt es schließlich Betriebe, in denen die ERAUmsetzung im Grundzug konflikthaft erfolgt (Typ IV, drei Betriebe). Einführungsprozess, Arbeitsbeschreibungen und Eingruppierungen sind Felder einer in hohem Maße politisierten Auseinandersetzung, bei der es im Kern darum geht, die eigene Verhandlungsposition zu stärken und sich in der betrieblichen Öffentlichkeit auf Kosten der Gegenseite zu profilieren. Beide Seiten versuchen, Informationsvorteile zur Durchsetzung der eigenen Interessen zu nutzen, und der Umgang mit den Regeln des Tarifvertrags erfolgt instrumentellstrategisch. Im Mittelpunkt der Arbeitsweisen und (Ver-)Handlungsstrategien stehen die Legitimierung der eigenen Position und die Delegitimierung der Gegenseite.

\subsection{RAHMENBEDINGUNGEN UND GRUNDLINIEN DER UMSETZUNG}

Modifiziert wird die im Kern in den vier Typen unterschiedlich ausgerichtete und verlaufende ERA-Umsetzung durch weitere Faktoren bzw. Rahmenbedingungen, die jedoch eher fallweise wirken. Die wirtschaftliche Lage des Unternehmens (der in 
einigen Fällen Standortsicherungsabkommen im Zusammenhang mit der ERA-Einführung Rechnung tragen), belegschaftsstrukturelle Besonderheiten und bislang vorherrschende Eingruppierungspraktiken (die in einigen Fällen eine erhebliche positive Lohndrift bewirkt haben) oder auch die Betriebsform (Konzernbetriebe oder Eigentümer-Betriebe) prägen Sichtweisen und Vorgehensweisen bei der ERA-Umsetzung in unterschiedlicher Weise und führen im Einzelfall zu Verzögerungen, Blockaden und Konflikten. Haupttendenz der ERA-Umsetzung im Tarifgebiet Niedersachsen sind bisher jedoch Variationen eines Grundmusters der Konfliktpartnerschaft. ${ }^{3}$ Unterschiedliche Interessen werden als legitim angesehen, ein betrieblich vermittelbarer und tragfähiger Kompromiss gilt dennoch als gemeinsam geteilte Zielperspektive. Angestrebt wird eine sachlich-fachlich korrekte Umsetzung des Tarifvertrags, die aber pragmatische Anpassungen an betriebliche Gegebenheiten nicht ausschließt.

Unter Beteiligungsgesichtspunkten Beteiligung und Mitgliederbindung haben programmatisch in der IG Metall durchaus eine Rolle gespielt - stellen die ERA-Einführungsprozesse nur in einer Minderheit der Betriebe eine Veränderung der eher repräsentativen Interessenvertretungspraxis dar. Eine systematische, breitflächige Einbeziehung der Belegschaft beim zentralen Thema Eingruppierung ist nur in wenigen Betrieben erfolgt. ${ }^{4}$ Verbreitet sind Vorgehensweisen, bei denen Beschäftigte eher fallweise aufgefordert wurden, Arbeitsplatzbeschreibungen zu überprüfen. Ein Beteiligungsschub hat in der großen Mehrheit der Betriebe nicht stattgefunden. Der notwendige zeitliche Aufwand und die Komplexität sowie Brisanz der Thematik haben vielfach eher dazu geführt, dass der Einführungsprozess auf beiden Seiten in den Händen einer überschaubaren Gruppe betrieblicher Experten lag.

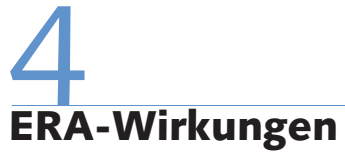

Zwar ist ERA im Tarifgebiet Niedersachsen im Herbst 2008 erst in der Hälfte der knapp 200 Betriebe eingeführt, und es ist nicht auszuschließen, dass die Späteinführer in höherem Maße mit besonderen Bedingungen (höhere Kostensteigerungen oder grö-

\section{Tabelle 1: Beschäftigte mit Überleitungszulage (Überschreiteranteile)}

\begin{tabular}{lcc}
\hline $\begin{array}{l}\text { Überleitungszulagen (ÜLZ): } \\
\text { Beschäftigtenanteile in \% }\end{array}$ & $\begin{array}{c}\text { Fallstudien } \\
\text { (Betriebe) }\end{array}$ & $\begin{array}{c}\text { Rückmeldebögen } \\
\text { (53 Betriebe) in \% }\end{array}$ \\
\hline 0-19 ÜLZ-Anteil & 6 & 25 \\
20-49 ÜLZ-Anteil & 5 & 40 \\
50-79 ÜLZ-Anteil & 2 & 23 \\
80-100 ÜLZ-Anteil & 2 & 13 \\
\hline & & WSI MITTEILUNGEN \\
Quelle: Fallstudien SOFI; Erhebung IG Metall; Berechnungen der Autoren. & &
\end{tabular}

ßere Konflikte) konfrontiert sein werden. Auf der Basis der Fallstudien, Betriebsdaten und Experteneinschätzungen lassen sich aber dennoch bereits einige Grundzüge der ERA-Wirkungen umreißen.

\subsection{GROßE SPANNBREITE BEI ÜBERSCHREITERANTEILEN}

Auch wenn tarifvertraglich geregelt ist, dass durch ERA keine individuellen Einkommensnachteile entstehen, spielt die sogenannte „Überschreiter-Frage“ (Personen mit Überleitungszulage) in den Betrieben eine erhebliche Rolle. Ungeachtet der tarifdynamischen Absicherung werden Überleitungszulagen von den Beschäftigten als Ausdruck eines Verlustes an betrieblicher Anerkennung und Zeichen verringerter Wertschätzung der eigenen Arbeit erlebt. Teile des Einkommens gelten mit einem Male als „nur noch zugestanden“, aber nicht mehr als ,verdient“. Vor diesem Hintergrund erstaunt es daher auch nicht, dass der Anteil von Personen mit Überleitungszulagen in hohem Maße für das Ausmaß an Unruhe in den Betrieben verantwortlich ist.

Bei den Überschreiteranteilen lassen sich zwischen den Betrieben erhebliche Unterschiede feststellen (Tabelle 1). In den Fallstudienbetrieben finden sich nicht wenige Betriebe mit Quoten, die unter $20 \%$ liegen, es gibt jedoch auch Fälle, in denen über $80 \%$ der Beschäftigten eine Überleitungszulage erhalten. In gut einem Drittel der Betriebe, aus denen Daten vorliegen, übersteigt der Überschreiteranteil $50 \%$. In den jeweiligen Quoten schlagen sich verschiedene Einflussfaktoren sowie spezifische betriebliche Konstellationen nieder. Wobei neben der aktuellen wirtschaftlichen Situation und der Bedeutung von Personalkostenreduktionsstrategien bei der ERA-Einführung vor allem das Ausmaß eine Rolle spielt, in dem in früheren Zeiten betriebliche Lohnpolitik betrieben wurde, die zu einer positiven Lohndrift geführt hat. Besonders niedrige Anteile von Be- schäftigten mit Überleitungszulagen ergeben sich in Betrieben typischerweise dann, wenn die arbeitsmarktbedingte betriebliche Lohndrift gering ausgeprägt war, teilweise in Betrieben mit geringem Angestelltenanteil sowie bei besonders guter wirtschaftlicher Lage bzw. expandierenden Betrieben.

Da sich in der Frage der Eingruppierung sehr unterschiedliche betriebsstrukturelle, historische und strategische Einflussfaktoren überlagern, lassen sich die Überschreiteranteile kaum auf einzelne Konstellationen zurückführen. Erwartungskonform ist allerdings der Befund, dass es einen engen Zusammenhang zwischen den Typen der ERA-Umsetzung und dem Anteil von Überleitungszulagen gibt (Abbildung 1): Betriebe mit hohen Anteilen von Überleitungszulagen gehören fast durchweg zu den Typen III und IV (begrenzte Konflikte, konflikthafte ERA-Umsetzung).

3 Bezogen auf die 22 durchgeführten Kurzfallstudien lässt sich dieser Befund insofern konkretisieren, als bei der Mehrheit der Betriebe der Umfang von Reklamationen unter $10 \%$ lag und in nur fünf Betrieben eine Quote von mindestens einem Drittel erreicht wurde. Ein ähnliches Bild einer geringen Zahl von Reklamationen ergibt sich auch auf der Grundlage von Daten, die auf Rückmeldungen von Betriebsräten an die Bezirksleitung der IG Metall basieren: Knapp $30 \%$ der Betriebe hatte keine Konfliktfälle, in über zwei Dritteln der Betriebe lag die Quote bei bis zu $10 \%$.

4 Die Beteiligung der Beschäftigten durch den Betriebsrat im Prozess der Eingruppierung vor Bekanntgabe des Ergebnisses reicht von kompletter Nichtbeteiligung über persönliche Ansprache in klärungsbedürftigen Einzelfällen bis hin zu systematischer Ansprache und/oder der Aufforderung, eigene Arbeitsbeschreibungen zu erstellen. Bei der Beteiligung von Bereichsleitern erstreckt sich die Bandbreite von Beispielen, in denen Arbeitsbeschreibungen und Eingruppierung von diesen komplett selbst erstellt wurden, bis hin zu Fällen, in denen vom Personalbereich erstellte Beschreibungen und Eingruppierungen lediglich gegengelesen und abgeglichen wurden. Eher die Ausnahme - und eher in kleineren Abteilungen sowie in Angestelltenbereichen anzutreffen - sind Fälle, in denen die Bereichsleiter ihrerseits die Beschäftigten einbezogen haben. 


\section{Abb. 1: Beschäftigte mit Überleitungszulage nach Umsetzungstypen (Überschreiter) - in \% -}

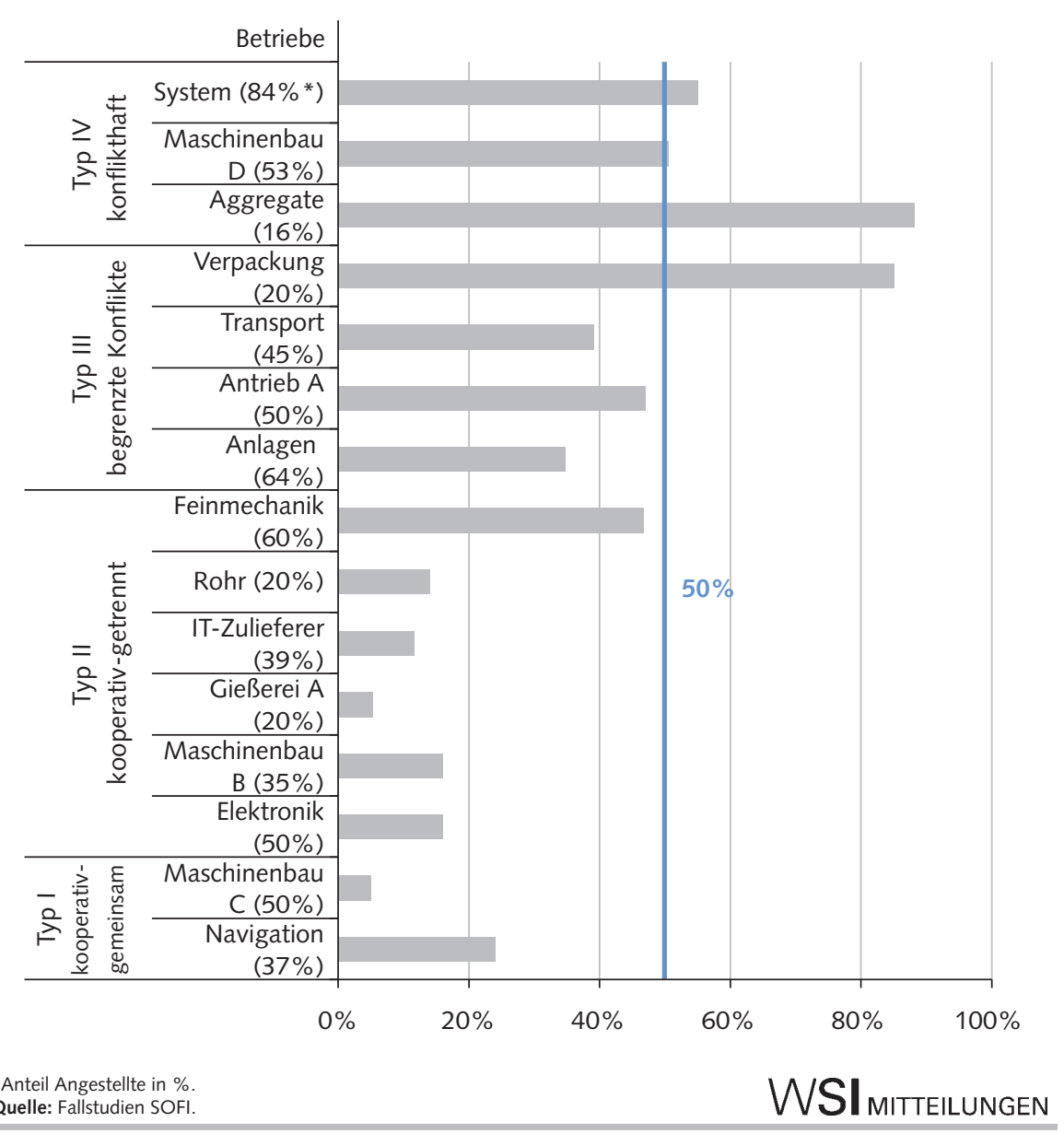

lierer"), deutliche und vergleichsweise einheitliche Muster feststellen. ${ }^{5}$ Je nach konkreter betrieblicher Situation und bisheriger Eingruppierungspraxis können Tätigkeitsbereiche oder Personengruppen zwar unterschiedlich betroffen sein, im Kern finden sich jedoch wiederkehrende Einflussfaktoren.

Aus tarifsystematischen Gründen entstehen niedrigere Eingruppierungen bzw. Überleitungszulagen insbesondere bei mittleren Angestellten: teils weil berufserfahrungsbedingte Gehaltsstufensprünge entfallen, häufiger noch jedoch deshalb, weil mittlere Angestellte aufgrund der geringen Anzahl von Gehaltsgruppen im alten System besonders hoch eingruppiert waren (Tabelle 2). Effekte, die sich aus der bisherigen Eingruppierungspraxis ergeben, spielen insgesamt eine große Rolle. Beschäftigte mit Überleitungszulagen finden sich in der Hauptsache in Tätigkeitsbereichen bzw. bei Personengruppen, die aus unterschiedlichen Gründen (arbeitsmarktbedingt, besonderer betrieblicher Stellenwert) bisher besonders gut eingruppiert waren. Aufgrund der Ausrichtung der Entgeltgruppen an Anforderungsniveaus, die mit formalen Qualifikationsniveaus einhergehen (Meister-, Techniker-, Fachhochschulniveau), kommt es gerade im mittleren Bereich gehäuft zu Überleitungszulagen.

Von tarifsystematischen Effekten und Folgewirkungen historisch gewachsener Eingruppierungspraktiken zu unterscheiden sind Überleitungszulagen, die sich eher als Folge technologischen Wandels sowie veränderter Ausbildungsinhalte erklären lassen. D.h. sie greifen dann, wenn die Arbeitstätigkeit im Zuge veränderter Rahmenbedingungen geringere formale Qualifikationen erfordert. Eher unspezifisch, quantitativ aber sehr wohl bedeutsam, sind schließlich Überleitungszulagen, die daraus resultieren, dass individuelle Tätigkeitswechsel auf geringerwertige Arbeitsplätze in der Bezahlung bisher nicht nachvollzogen wurden.

Diesem Bündel von Verursachungszusammenhängen für niedrigere Eingruppie-

\footnotetext{
5 In finanzieller Hinsicht erleidet die hier als „Verlierer" bezeichnete Personengruppe aufgrund der Überleitungszulage keinen Verlust, die betrieblichen Entgelte werden sogar tarifvertraglich abgesichert. Wir folgen jedoch den Situationsdeutungen und Selbst-/Fremdwahrnehmungen in unserem Untersuchungsfeld, bei denen die Aspekte fehlender Anerkennung und Unsicherheitsgefühle eine erhebliche Rolle spielen.
}

Während die Anteile von Beschäftigten mit stark differieren, lassen sich bei der Frage, welche Tätigkeiten bzw. Personengruppen eher mit höheren Tarifentgelten zu rechnen haben („Gewinner") und wo typischerweise Überleitungszulagen entstehen („Ver- 
rungen stehen auf der anderen Seite nur wenige Konstellationen gegenüber, die bei ERA zu einer höheren Bezahlung oder gar höheren Eingruppierung führen und bei denen es sich zumeist um Effekte der Durchsetzung des Gleichbehandlungsgrundsatzes handelt. Quantitativ bedeutsam ist vor allem die Gruppe der bisherigen Zeitlöhner, bei denen sich höhere ERAEntgelte tarifsystematisch aufgrund der gerade im Facharbeiterbereich deutlich angehobenen Grundentgelte ergeben. Ferner profitieren vor allem jüngere Beschäftigte, insbesondere im Angestelltenbereich. Diese Gruppe konnte altersbedingt an den Senioritätsvorteilen, die das alte Tarifsystem geboten hatte, ohnehin nicht partizipieren. Die ERA-Einführung bedeutet für sie eine sofortige Höhergruppierung. Gleiches gilt für weibliche Beschäftigte, vor allem für Produktionsarbeiterinnen, - zumindest dann, wenn es vor ERA Frauen benachteiligende Lohndifferenzen im Vergleich zu männlichen Beschäftigten mit ähnlichen Tätigkeiten gab. Gleichwohl dürfte die „Gewinner/Verlierer-Bilanz“ unter Gendergesichtspunkten für Frauen bei ERA eher negativ ausfallen, weil in der Metall- und Elektroindustrie die Anteile von Frauen in der Produktion bzw. in gemischt-geschlechtlichen Arbeitsbereichen ungleich geringer sind als die der Frauen, die in den durch ERA heruntergestuften Bereichen arbeiten: als mittlere, kaufmännische Angestellte im Sekretariatsbereich oder als technische Zeichnerin.

Verglichen mit ersten vorliegenden Befunden aus anderen Tarifgebieten scheinen sich für das Tarifgebiet Niedersachsen recht ähnliche Gewinner- und Verlierergruppen herauszubilden. Im Abgleich mit vorliegenden Daten aus Baden-Württemberg bewegen sich zudem die aggregierten Effekte für Über- und Unterschreiter in vergleichbaren Größenordnungen: Der derzeit etwas geringeren Überleitungszulagenquote von $43 \%$ in Niedersachsen steht ein Überschreiteranteil von $54 \%$ in der badenwürttembergischen Befragung gegenüber (vgl. Bahnmüller/Schmidt in diesem Heft). Ein ebenfalls über die verschiedenen Tarifgebiete hinweg ähnliches Ergebnis ist, dass die durchschnittlichen Euro-Beträge der Überleitungszulagen über den Entgeltzuwächsen durch ERA liegen. Während immerhin $17 \%$ der Beschäftigten Überleitungszulagen von über $200 €$ haben, liegt der Anteil von Entgeltsteigerungen über $200 €$ nur bei gut $10 \%$ (Abbildung 2).

\section{Abb. 2: Differenzbeträge beim Entgelt - in Euro -}

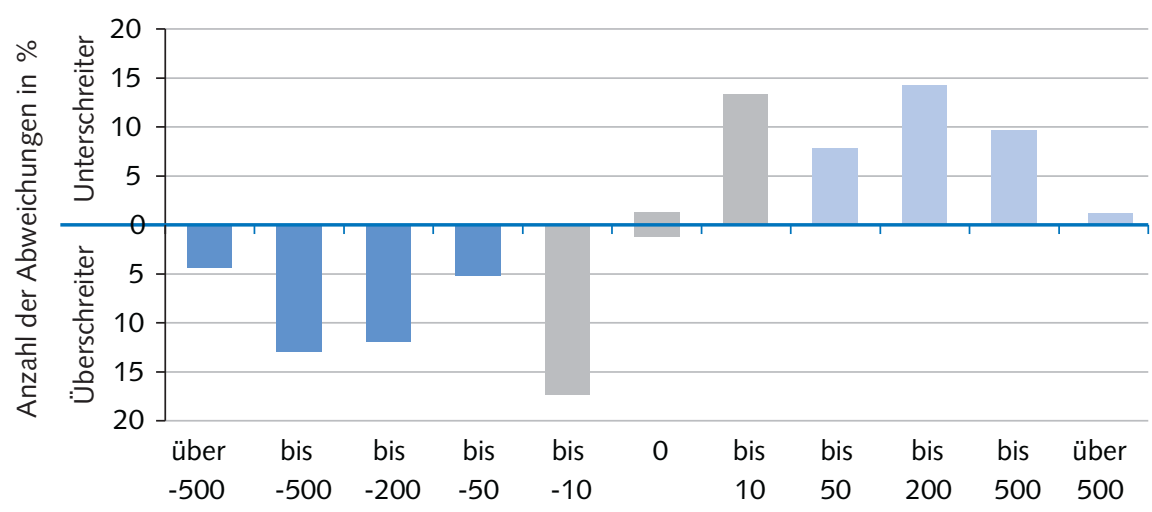

Höhe der Abweichungen in $€$ (Überschreiter, Unterschreiter)

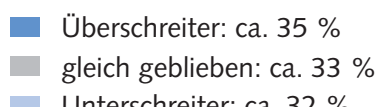

Quelle: Fallstudien SOFI.

WSI MITTELUNGEN

\begin{tabular}{lcc}
\hline Tabelle 3: Tarifliche Mehrkosten durch ERA & \\
\hline Zunahme der Tarifentgeltsumme in \% & $\begin{array}{c}\text { Fallstudien } \\
\text { (Anzahl Betriebe) }\end{array}$ & $\begin{array}{c}\text { Rückmeldedaten } \\
\text { (n=46 Betriebe) in \% }\end{array}$ \\
\hline $0-1$ & 4 & 24 \\
über 1 bis unter 2 & 5 & 35 \\
$2-2,7$ & 2 & 15 \\
ca. 2,79 & 4 & 9 \\
über 2,79 & 2 & 17 \\
\hline & & WSI MITTEILUNGEN \\
Quelle: Fallstudien SOFl; Erhebung IC Metall; Berechnungen der Autoren. &
\end{tabular}

\subsection{MATERIELLE WIRKUNGEN BILANZIERT}

Auch im Tarifgebiet Niedersachsen gilt, dass Kostenneutralität laut Definition des Tarifvertrags bei betrieblichen Mehrkosten von $2,79 \%$ gegeben ist. $^{6}$ Auffällig ist bei den Tarifentgelten als wichtigem Indikator der materiellen Wirkungen von ERA zunächst wiederum die große Spannbreite (Tabelle 3). Nur eine Minderheit der Betriebe erreicht oder überschreitet eine Erhöhung der tariflichen Entgeltsumme um 2,79\%. Dem stehen auf der anderen Seite Betriebe gegenüber, die teils aufgrund von Standortsicherungsvereinbarungen keine oder allenfalls eine geringe Steigerung der Tarifentgeltsumme verzeichnen. Auch unter der Annahme, dass im weiteren Verlauf der ERA-Einführung die Quote der Betriebe mit Kosten über 2,79 \% noch steigt, dürfte bezogen auf das Tarifgebiet insgesamt eine Quote von 2,79 \% deutlich unterschritten werden - sowohl nach Betrieben gerechnet als auch nach Beschäftigten. Anders als bei der Frage der Überschreiterquoten sprechen unsere Befunde bislang nicht für einen Zusammenhang zwischen den Umsetzungstypen und den materiellen Ergebnissen beim Entgeltvolumen (Abbildung 3).

Verteilungspolitisch fügt sich somit auch die ERA-Bilanz in die seit einer ganzen Reihe von Jahren konstatierte Tendenz einer moderaten Lohnpolitik ein (Bispinck 2007). Wie sich ERA in materieller Hinsicht auf Dauer auswirkt, ist derzeit nicht sicher einzuschätzen. Zunächst einmal lassen sich die Überleitungszulagen potenziell jedoch als von den Betrieben mittel- und langfristig einzusparende Personalkosten interpretieren. Sobald Beschäftigte aus dem Betrieb ausscheiden, entfallen die Überleitungszulagen für nachrückende Personen; niedrigere Eingruppierungen der ERA-Einführung werden dann entgeltwirksam. Ob sich die mit diesem Kalkül

\footnotetext{
6 Im Tarifgebiet Niedersachsen bezieht sich der Wert auf die Erhöhung der Tarifentgeltsumme einschließlich der Überleitungszulagen. In genau diesem Umfang waren Tariferhöhungen in den Jahren 2002 bis 2005 nicht tabellenwirksam geworden.
} 
Abb. 3: Tarifliche Mehrkosten - in \% -

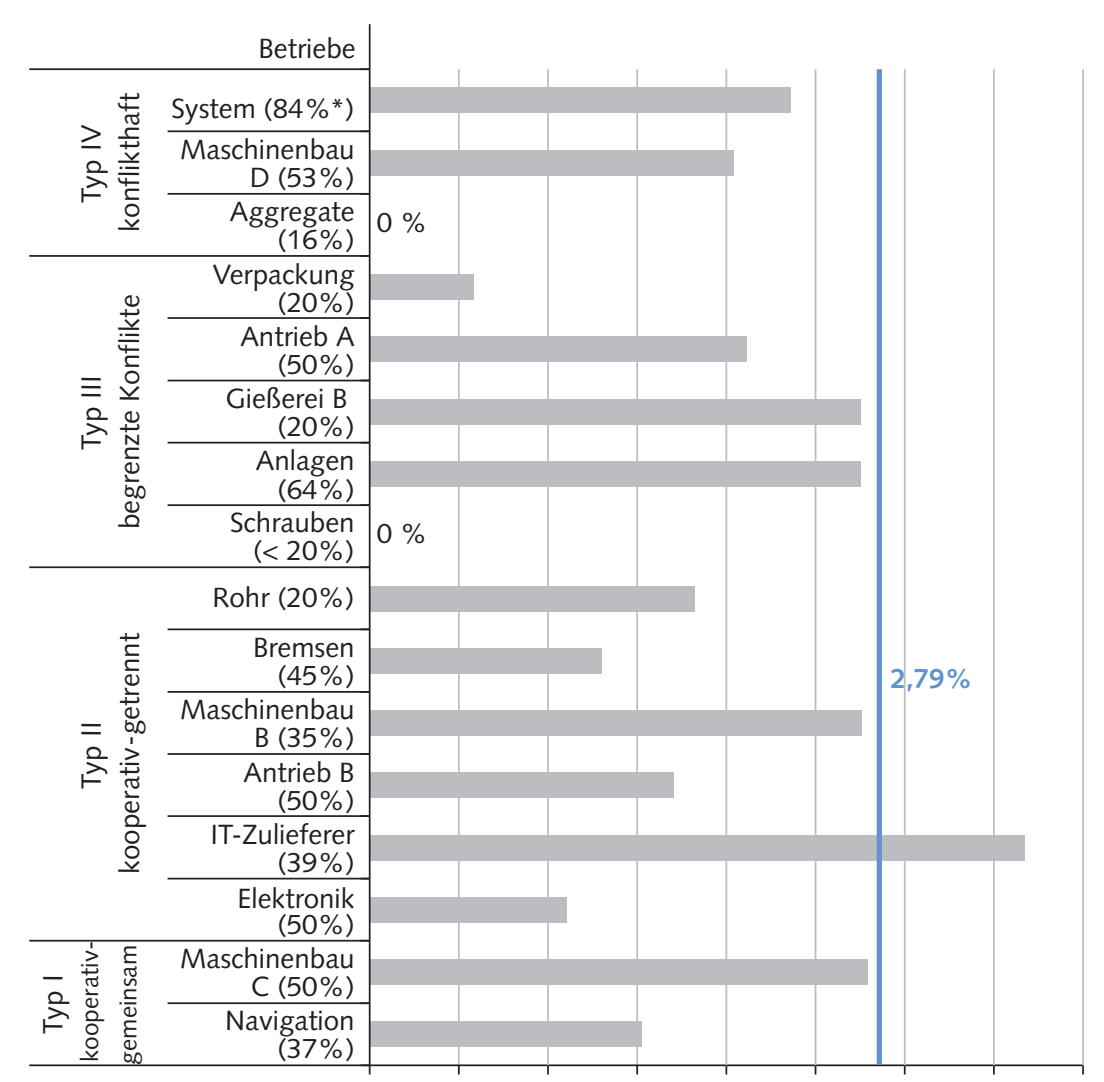

$\begin{array}{lllllllll}0,0 \% & 0,5 \% & 1,0 \% & 1,5 \% & 2,0 \% & 2,5 \% & 3,0 \% & 3,5 \% & 4,0 \%\end{array}$

angestrebten Personalkosteneinsparungen wirklich realisieren lassen, bleibt allerdings abzuwarten.

\subsection{QUALITATIVE ERA-WIRKUNGEN}

Während sich bei den materiellen ERAWirkungen - bezogen auf die Entgeltlinie insgesamt wie auf die Betroffenheit von Beschäftigtengruppen - stark differenzierte Effekte nachweisen lassen, ist die Ausgestaltung der Entgeltgrundsätze und Entgeltsysteme durch ein hohes Maß an Kontinuität geprägt.7 Knapp zwei Drittel der Beschäftigten hatten Zeitlohn bzw. Gehalt vor ERA und haben jetzt Zeitentgelt; der Prämienlohn bzw. Prämienentgeltanteil liegt bei gut einem Drittel. Akkordentgelt ist fast vollständig verschwunden. Die Gehaltsempfänger und die bisherigen Zeitlöhner werden bei ERA ganz überwiegend in den Grundsatz Zeitentgelt überführt. Wechsel des Entgeltgrundsatzes (von Zeitlohn/Gehalt ins Leistungsentgelt oder vom Leistungslohn ins Zeitentgelt) bleiben bislang die Ausnahme. Das neuerdings tarifvertraglich geregelte Zielentgelt hat im
Tarifgebiet Niedersachsen bisher nahezu keine Verbreitung gefunden. Zielvereinbarungen sind insbesondere in Angestelltenbereichen in einigen Betrieben zwar mittlerweile Alltag, allerdings nicht als Grundlage für Entgeltbestandteile. Bei der Überführung vorhandener Entlohnungssysteme in ERA wird zumeist das im Tarifvertrag ohnehin vorgesehene Verfahren der redaktionellen Anpassung bestehender Regelungen praktiziert. Dies muss gleichwohl nicht heißen, dass auch in Zukunft bei Entgeltgrundsätzen und Entgeltsystemen alles beim Alten bleibt. In einer Reihe von Betrieben wurde in den Gesprächen sogar betont, dass man die bisherigen Entgeltsysteme nur im ersten Schritt nicht geändert habe, für die Zukunft aber durchaus Änderungen angedacht oder bereits im Gespräch seien. Da die Vorstellungen in vielen Fällen allerdings überaus vage sind und von den Betriebsparteien nicht selten unterschiedliche Zielsetzungen geäußert wurden, bleibt abzuwarten, inwieweit es zukünftig, durch ERA angestoßen, wirklich zu einer breitflächigen Durchsetzung neuer Entgeltsysteme kommt. Feste Verabredungen zwi- schen den Betriebsparteien sowie prinzipiell einheitliche Vorstellungen über künftige Systeme gibt es bisher nur vereinzelt.

Gleichwohl bedeutet diese eher skeptische Einschätzung der qualitativen ERAWirkungen nicht, dass in den von uns untersuchten Betrieben keinerlei Bewegung in der Frage der Entgeltsysteme zu beobachten wäre. In einem Teil unserer Fallstudienbetriebe hat es seit den 1990er Jahren sehr wohl Veränderungen gegeben. Diese werden bei ERA fortgeschrieben; in einzelnen Fällen ist es im Umfeld der ERAEinführung, teils im Vorgriff, teils nachgelagert, auch zu einer beschleunigten Verbreitung von seit Längerem eingeleiteten Entgeltsystemveränderungen gekommen. Verdichtet man die in einzelnen Betrieben zu beobachtenden Trends und die in den Interviews formulierten Zielvorstellungen zu Tendenzaussagen, dann lassen sich - allerdings mit der gebotenen Vorsicht durchaus ein paar typische, wenn auch in der Fläche eher schwach ausgeprägte Entwicklungslinien unterscheiden. So werden beispielsweise stückzahlbasierte Leistungslohnsysteme auf neue Prämiengrößen umgestellt und Prämienentgeltsysteme ausgeweitet, die sich an neuartigen Leistungsmerkmalen wie Liefertreue, Lagerbestände oder Qualitätskennziffern ausrichten. Andererseits dürfte der bislang hohe Zeitentgeltanteil kaum sinken und könnte, durch beschäftigungsstrukturelle Umschichtungen hin zu Dienstleistungstätigkeiten verstärkt, sogar eher noch ansteigen. Gerade Klein- und Mittelbetriebe scheuen nicht selten den für Leistungsentgeltsysteme notwendigen organisatorischen Aufwand und ziehen auch aus diesem Grund die Flexibilitätsvorteile des Zeitentgelts vor. Während Zielentgelt nach wie vor eher für Angestelltentätigkeiten diskutiert wird und sich insgesamt zumeist im Ideenstadium befindet, gibt es in einigen Betrieben Bestrebungen, zumindest produktionsnahe Angestelltentätigkeiten in Prämienentgeltsysteme einzubeziehen. Bei den Leistungsbeurteilungen im Zeitentgelt befinden sich formalisierte Beurteilungssysteme bislang noch deutlich in der Minderheit. Zudem spielen gleichmäßige Verteilungen von Leistungs-

\footnotetext{
Auch arbeitspolitische Effekte sind bei ERA bislang nicht zu beobachten oder allenfalls schwach ausgeprägt. Charakteristisch ist vielmehr, dass ERA bestehende Tendenzen der Re-Taylorisierung oder Höherqualifizierung festschreibt oder allenfalls als Verstärker ohnehin vorhandener Tendenzen wirkt.
} 
zulagen nach wie vor eine große Rolle. Bei den Beurteilungssystemen ist jedoch ein Trend erkennbar, der auf die Abkehr von rein subjektiven Verfahren („Nasenprämie“) abzielt und formalisierte Verfahren etabliert - zudem finden Mitarbeitergespräche eine stärkere Verbreitung. Letztlich bestätigen die bisherigen Befunde der ERAUmsetzung das Bild früherer Untersuchungen, dass neue Entgeltsysteme bislang nur in einer Minderheit von Betrieben im Einsatz sind und auch bei deren Ausbreitung keineswegs mit sprunghaft steigenden Zahlen zu rechnen ist (Schmierl 1996; Lay/ Rainfurt 1999; Bahnmüller 2001).

\section{(Zwischen-)Bilanz}

Auch wenn es angesichts des Einführungsstandes und der bislang durchgeführten Erhebungen noch zu früh ist, die ERA-Umsetzung im Tarifgebiet Niedersachsen abschließend zu bilanzieren, deuten sich schon jetzt einige wichtige Befunde an. Im Vergleich mit den bereits vorliegenden Untersuchungsergebnissen zu Baden-Württemberg (Bahnmüller/Schmidt 2007, 2008 sowie dieselben in diesem Heft) fällt ins Auge, dass die Unterschiede im Bereich der materiellen Wirkungen (Betroffenheit der verschiedenen Beschäftigtengruppen und Tätigkeitsbereiche, Überschreiterquoten, betriebliche Kosten, Verteilungsbilanz) zumeist eher gering sind, ${ }^{8}$ in der Frage des Einführungsprozesses jedoch erheblich. In beiden Tarifgebieten stellt ERA einen politisch brisanten und überaus arbeits- und verhandlungsaufwendigen Prozess dar, der in seinen materiellen Wirkungen von den Tarif- und Betriebsparteien vielfach unterschätzt wurde. Während der Einführungsprozess in Baden-Württemberg in einer ganzen Reihe von Betrieben erhebliche Auseinandersetzungen produzierte und nicht zuletzt mit Konflikten auf der Ebene der Verbände einherging, steht der „Niedersachsen-Weg" für eine konfliktdämpfende, ein hohes Maß an Kooperation auf der betrieblichen Ebene ermöglichende Umsetzungsstrategie. Die Strategien der Tarifvertragsparteien sowie die Ausgestaltung der Tarifregeln selbst basieren auf Orientierungen einer kooperativen Konfliktverarbeitung. Die ERA-Umsetzung wurde ausdrücklich nicht als betriebliche Verteilungsauseinandersetzung betrieben. Der Verzicht auf Kontinuitätsbrüche und die Absicherungskomponenten unterstützen eine pragmatische, tarifkonforme Anpassung der Entgeltstrukturen, vermeiden aber gleichzeitig, dass ERA als ein technischer Vorgang weitgehend entpolitisierter Regelanwendung von den Betriebsakteuren lediglich exekutiert wird. Der tarifliche Gestaltungsanspruch wurde von den Betriebsakteuren aufgegriffen, und der Flächentarifvertrag hat an Regulierungskraft gewonnen, wobei den betrieblichen Gegebenheiten ausdrücklich Rechnung getragen wird und der Spielraum der Betriebe insbesondere in der Frage der Gestaltung der Entgeltsysteme groß ist und sich tendenziell erweitert. Die Notwendigkeit, die Handlungsfähigkeit der betrieblichen Akteure zu sichern, wird von beiden Seiten gesehen: Der regionale Arbeitgeberverband NiedersachsenMetall betont zwar die Ordnungsfunktion von Tarifverträgen, formuliert anders als Südwestmetall gegenüber den Betrieben jedoch keinen starken Gestaltungsanspruch. Er agiert eher in der Rolle eines Dienstleisters und weniger als Treiber. Mit diesem Rollenverständnis folgt er nicht nur der bisherigen regionalen Tariftradition, sondern entspricht beim Thema ERA auch den Erwartungen der Betriebe. Die IG Metall stellt den Betriebsräten in erheblichem Maße Informationen und Schulungen zur Verfügung und liefert damit praktisches Orientierungswissen, ohne dabei ihrerseits jedoch eine Handlungsho- heit beanspruchen zu wollen oder zu können. Von den Tarifparteien in Niedersachsen wird das ERA-Reformprojekt deshalb als Zeichen einer Stabilisierung des Flächentarifvertrags gewertet und nicht als Pfadwechsel wahrgenommen.

Im Ergebnis lässt sich die ERA-Einführung also eher als Beleg für die fortbestehende Gestaltungskraft des Flächentarifvertrags interpretieren und nicht für dessen Bedeutungsverlust, Aushöhlung oder Umnutzung. ERA ist bei den beteiligten Akteuren im Großen und Ganzen akzeptiert: auf Arbeitgeber- wie Gewerkschaftsseite hat es in nennenswertem Umfang weder Austritte noch Eintritte gegeben. Die Umsetzungsverläufe von ERA in Niedersachsen haben die Verpflichtungsfähigkeit der Tarifparteien erwiesen; die betriebliche Praxis bei Eingruppierungsfragen und Entgeltsystemen ist wieder stärker durch Tarifnormen geprägt. ERA fügt sich zugleich in eine allgemeine Tendenz ein, der zufolge Tarifverträge über verbindliche Normen hinaus zunehmend Optionen bereitstellen und Prozessregelungen formulieren, die von den betrieblichen Akteuren ausgefüllt werden müssen. Die betriebliche Interessenvertretung ist immer weniger (nur) tarifpolitischer Exekutor, sondern wird in hohem Maße zu einer eigenständigen Akteursebene. Insofern stellt auch das Tarifreformprojekt ERA neue Herausforderungen an die Austarierung des Verhältnisses von tariflicher und betrieblicher Ebene, die über den Zeitrahmen der ERA-Einführung hinausreichen.

8 Eine Ausnahme stellt hierbei der in Baden-Württemberg deutlich gestiegene, in Teilen aber auch aus dem veränderten Umgang mit Belastungen resultierende Anteil von Eingruppierungen unterhalb des Eckentgelts dar. Im Tarifgebiet Niedersachsen scheint diese Quote weniger stark gestiegen zu sein. 


\section{LITERATUR}

Bahnmüller, R. (2001): Stabilität und Wandel der Entlohnungsformen. Entgeltsysteme und Entgeltpolitik in der Metallindustrie, in der Textil- und Bekleidungsindustrie und im Bankgewerbe, München/Mering

Bahnmüller, R./Schmidt, W. (2007): Auf halbem Weg. Erste Befunde zur ERA-Umsetzung in Baden-Württemberg, in: WSI-Mitteilungen 7, S. 358364

Bahnmüller, R./Schmidt, W. (2008):Der ERA und seine Umsetzung. Erfahrungen aus Baden-Württemberg, in: Bispinck, R. (Hrsg.): Verteilungskämpfe und Modernisierung. Aktuelle Entwicklungen in der Tarifpolitik, Hamburg, S. 78-118

Beraus, W. (2004): Neues regeln - Bewährtes erhalten - Beteiligungsrechte stärken: Der Entgeltrahmentarifvertrag in Baden-Württemberg, in: WSI-Mitteilungen 2, S. 112-114

Bispinck, R. (2007): Löhne, Tarifverhandlungen und Tarifsystem in Deutschland 1995-2005, WSI-Diskussionspapier 150, Düsseldorf Brunkhorst, C./Burkhard, O./Scherbaum, M. (Hrsg.) (2006): Eine neue AERA. Tarifverträge für die Zukunft, Hamburg

Huber, B./Schild, A. (2004): Die neuen Entgeltrahmentarifverträge, in: WSI-Mitteilungen 2, S. 102-105

Huber, B./Burkhard, O./Wagner, H. (Hrsg.) (2006): Perspektiven der Tarifpolitik, Hamburg

Lay, G./Rainfurt, C. (1999): Königsweg "Prämie“?, Mitteilungen aus der Produktinnovationserhebung 13, PI-Mitteilungen, FhG-ISI, Karlsruhe
Manthey, M./Meine, H. (2004): Der neue Entgeltrahmentarifvertrag in der niedersächsischen Metallindustrie, in: WSI-Mitteilungen 2, S. 108109

Reichel, F.-G. (2005): Das Regelungswerk ERA zur Neugestaltung der tariflichen Vergütung in der Metall- und Elektro-Industrie, in: Angewandte Arbeitswissenschaft 183, S. 2-26

Reichel, F.-G./Hofmann, A./Gryglewski, S./Bossemeyer, A. (2005): ERA. Der neue Entgeltrahmen der Metall- und Elektroindustrie, Leistung und Lohn 405-409, Berlin

Sadowsky, R. (2004): Der Entgeltrahmentarifvertrag in NRW: was lange währt..., in: WSI-Mitteilungen 2, S. 110-112

Schmierl, K. (1996): Historische Leistungskompromisse unter Veränderungsdruck - Neue Lohnsysteme in der Metallindustrie, in: WSI-Mitteilungen 10, S. 652-660

Schulz, H. (2004): Gute Arbeit - neue AERA - Der Entgeltrahmentarifvertrag im Norden, in: WSI-Mitteilungen 2, S. 105-107

Streeck, W./Rehder, B. (2003): Der Flächentarifvertrag: Krise, Stabilität und Wandel, in: Industrielle Beziehungen 3, S. 341-362

Weltz, F. (1977): Kooperative Konfliktverarbeitung. Ein Stil industrieller Beziehungen in deutschen Unternehmen, in: Gewerkschaftliche Monatshefte 5, S. 291-301 